\title{
Function Generation Synthesis of Spherical 5R Mechanism with Regional Spacing and Chebyshev Approximation
}

\author{
Gökhan Kiper ${ }^{1, a}$, Tunç Bilgincan ${ }^{\mathrm{a}}$
}

${ }^{a}$ Mechanical Engineering Department, İzmir Institute of Technology, 35430 İzmir, Turkey

\begin{abstract}
The Chebyshev approximation is well known to be applicable for the approximation of single input-single output functions by means of a function generator mechanism. The approximation method may be also applied to multi-input functions, although until recently, it was not used for function generation with multi-degrees-of-freedom mechanisms. In a recent study, the authors applied the approximation method to a two-degrees-of-freedom mechanism for the first time, however the selection and iteration of the design points at which the errors were minimized was not satisfactory. In this study, an alternative method of selection and iteration for these design points is introduced and the corresponding spacing is called the "regional spacing". As a case study for the application of the approximation of multi-input functions, a spherical 5R mechanism is used to generate a two input-single-output function. The input joints of the mechanism are selected as one of the fixed joints and the moving mid-joint, whereas the remaining fixed joint represents the output. The synthesis problem is analytically formulated and presented in polynomial form for five and six unknown parameters. The synthesis problem for five unknown parameters is illustrated as a numerical example. Regional spacing is used for the selection and iteration of design points for the synthesis. The Chebyshev approximation along with the Remez algorithm is utilized to find the unknown construction parameters and the error of the function. The design points and the coefficients of the approximation polynomial are determined by numerical iteration using six moving points. At each iteration step, the design points are relocated at the extremum error points in their respective regions. Iterations are repeated until the magnitudes of the extremum point errors are approximately equal. Finally, the construction parameters of the mechanism are determined and the variation of the percentage error between the desired and generated function values is obtained.
\end{abstract}

Keywords: function generation synthesis, Chebyshev approximation of bivariate functions, Remez algorithm, regional spacing, spherical five bar mechanism

\section{Introduction}

The well-known kinematic synthesis problems are path generation, body guidance and function generation. Although kinematic synthesis of single degree-of-freedom (dof) systems have been vastly studied, publications on synthesis of multi-dof systems are not very common. Svoboda [1] devised geometric means for designing planar mechanisms for simple two-input (bi-variate) functions such as addition, multiplication, and division of two numbers. Allen [2] made use of a graphical method for function generation synthesis with 2-dof linkages for a limited number of precision points. Philip and Freudenstein [3] worked on function generation with some of Svoboda's mechanisms by solving the loop closure equations numerically for 10 or 13 precision points. Lakshminarayana et al. [4-10] have worked on precision point and higher order synthesis of 7- and 9-link planar mechanisms to generate bi-variate functions. Mruthyunjaya [11-12] developed a graphical procedure based on point position reduction with six precision points. Kohli and Soni [13] worked on function/path and motion generation with 2-dof 7-link planar mechanisms by formulating the equations with displacement matrices and solving the set of equations numerically. For the function generation problem, they used a

\footnotetext{
${ }^{1}$ Corresponding author. Tel.: +90 232 7506777; fax: +90 2327506701.

E-mail address: gokhankiper@iyte.edu.tr (G. Kiper).
} 
2RRR-RR planar parallel mechanism with 13 precision points. Alizade et al. [14] formulized an unconstrained minimization problem for function generation, where the objective function was the sum of the squares of the errors at the selected design points on the two-dimensional input domain. The solution was found by an iterative numerical algorithm. Rao [15] combined a 2-dof planetary gear train with either a four-bar or a cam mechanism for function generation. Zhou and Cheung [16] designed a planar 5R (R: revolute joint) linkage using genetic algorithms. Lastly, Kim and Tsai [17] designed a 3dof 3-RPS parallel manipulator for rigid body guidance with six precision points in its workspace. In this study, the displacement equations between different poses of the platforms are written and the synthesis problem is formulized as a linear set of equations. The determinant of the coefficient matrix results in a maximum $10^{\text {th }}$ degree polynomial in terms of one of the link length parameters, which is solved numerically.

Most of the synthesis methods described above are based on the equations, where the functions are exactly generated at certain precision points in the domain of definition of the function. These methods are deemed interpolation methods. In the least squares approximation method, the aim is to minimize the sum of the squares of the errors at selected design points on the function domain. In [18-19], interpolation approximation is used for bi-variate function generation 2-dof linkages.

In both of interpolation and least squares approximation methods, the error between the desired function output and the output of the function generation mechanism (the structural error) are uncontrolled on the points on the domain other than the precision/design points. On the other hand, in the Chebyshev (Best) approximation method, the aim is to minimize the maximum absolute error on the whole function domain by equating the absolute error values at selected design points. Hence, if applicable, Chebyshev approximation appears superior than the other two methods for minimizing the maximum error. Interpolation, least squares and Chebyshev approximations methods were presented and used for the function generation problem of the planar four-bar linkage by Levitskii [20].

Although it is known that the Chebyshev approximation method can be also applied to multi-input functions [21-23], until recently, it has not been used for function generation with multi-degrees-offreedom mechanisms. An example of engineering applications is the design of two dimensional nonrecursive digital filters [24]. In a recent study [25], the authors applied the Chebyshev approximation method to a two-degrees-of-freedom mechanism for the first time. A planar five-bar mechanism is used and the five design points are chosen on the boundaries of the rectangular two-dimensional function domain. This type of selection is limited because the inside area of the function domain cannot be used. Selection of design points is one of the most important stages of approximation methods. For univariate function generation problems, it is known that the so-called Chebyshev spacing results in less error compared to other spacings [26]. For two-dimensional function domains, Freudenstein [27] has worked on optimum spacing for approximation with $(m \cdot n)^{\text {th }}$ degree polynomials and $m \cdot n$ many precision points. The optimum spacing was found to be Chebyshev spacing for both of the variable axes. However, this spacing proves useful if the approximation function is a polynomial.

In this study, we use a new type of selection for design points, which we call regional spacing. This type of spacing is superior to the other types of spacing alternatives we used before in that it allows a larger domain for the selection of design points and hence, increases the probability of obtaining a feasible solution with relatively less error.

As a case study of the application of Chebyshev approximation using regional spacing, we present the necessary formulation for a spherical $5 \mathrm{R}$ mechanism as a function generator. At the end of the study, a numerical example is presented, where iterative determination of the design points is performed starting with regional spacing and repeated until the extremum point errors are stabilized.

Kinematic synthesis with spherical mechanisms is widely studied. The function generation synthesis problem for spherical four-bar mechanisms for four precision points is studied by Zimmerman [28]. 
Computational methods for the exact kinematic synthesis of a spherical 4R mechanism for function generation synthesis problem are studied by Cervantes-Sánchez et al. [29]. Function synthesis with spherical 4R mechanisms for the five precision points is studied analytically by Alizade and Kilit [30]. Alizade and Gezgin [31] presented function generation synthesis of spherical four bar mechanism using quaternion algebra and introduced the comparison of interpolation, least squares, and Chebyshev approximations methods. A new algorithm is proposed by Peñunuri et al. for path generation problem of spherical 4R mechanism [32]. A novel optimization based methodology for synthesizing adjustable spherical four-link mechanisms is presented by Chanekar et al. [33]. Motion generation synthesis is studied with least square approximation method for spherical linkages by Alizade et al. [26]. A general theory for synthesis of spherical mechanisms is given in [34]. The reader is suggested to read Alizade et al. [26, 30, 31] for a more detailed review of design of spherical mechanisms.

\section{Chebyshev Approximation}

Polynomial approximation methods were first studied by Chebyshev in 1850 s $[35,36]$. Let $\mathrm{f}(\mathrm{x})$ be a continuous function defined on $\mathrm{x} \in[\mathrm{a}, \mathrm{b}]$. A polynomial $\mathrm{P}_{\mathrm{n}}(\mathrm{x})$ of degree $\mathrm{n}$ is called the best approximation of $\mathrm{f}(\mathrm{x})$ if $\mathrm{L}=\max \left|\mathrm{f}(\mathrm{x})-\mathrm{P}_{\mathrm{n}}(\mathrm{x})\right|$ is minimum. The alternation theorem of Chebyshev states that for a given function $\mathrm{f}(\mathrm{x})$ and order $\mathrm{n}$, the best approximation is unique and the maxima $\mathrm{L}$ are attained $n+1$ times on [a, b] alternately with opposite signs. Although this theorem guaranties the unique existence of the best approximation of a function, it does not lead to a method to find the best approximation. An iterative method is proposed by Remez [37]. The Remez algorithm is as follows [38]:

1. Select design points $x_{i}^{0} \in[a, b], i=1, . ., n+1$ (usually $x_{1}=a, x_{n+1}=b$ ) and linearly solve for the $n$ coefficients of an approximation polynomial $P_{n}{ }^{0}(x)$ and the error $L$ from the $n+1$ equations $\mathrm{P}_{\mathrm{n}}^{0}\left(\mathrm{x}_{\mathrm{i}}^{0}\right)-\mathrm{f}\left(\mathrm{x}_{\mathrm{i}}^{0}\right)=(-1)^{\mathrm{i}} \mathrm{L}$

2. Find the $n+1$ local extrema $x_{i}^{1}$ of $E^{0}(x)=P_{n}{ }^{0}\left(x_{i}^{0}\right)-f\left(x_{i}^{0}\right)$ in $[a, b]$

3. Repeat steps 1 and 2 by replacing $\mathrm{x}_{\mathrm{i}}{ }^{\mathrm{k}}$ by $\mathrm{x}_{\mathrm{i}}{ }^{\mathrm{k}+1}(\mathrm{k}=0,1, \ldots)$ until the design points stabilize

In function synthesis of mechanisms, we derive the input/output (I/O) relationship as a function of the construction parameters of the mechanism and input and output variables. The I/O equation is written in a polynomial form $P_{n}(\mathbf{x})-F\left(\mathbf{x}_{i}\right)=\sum_{j=1}^{n} P_{j} f_{j}(\mathbf{x})-F\left(\mathbf{x}_{i}\right)$, where $P_{j}$ are functions of the construction parameters, $f_{j}$ are the functions of the input and output variables represented by $\mathbf{x}$ and $\mathrm{F}(\mathbf{x})$ is the approximated function, called the objective function. If the number of coefficients $P_{j}=n$ is equal to the number of construction parameters, then given $n+1$ many design points $\left\{\mathbf{x}_{\mathrm{i}}\right\}_{1}^{\mathrm{n}+1}$, the coefficients $\left\{\mathrm{P}_{\mathrm{j}}\right\}_{1}^{\mathrm{n}}$ and the Chebyshev error L are linearly solved from

$$
\sum_{j=1}^{n} P_{j} f_{j}\left(\mathbf{x}_{i}\right)-F\left(\mathbf{x}_{i}\right)=(-1)^{i} L \text { for } \mathrm{i}=1, \ldots, n+1
$$

When the Chebyshev approximation is applied for single-variable functions, the sign of the error $\mathrm{L}$ is alternating for consecutive design points. However, in our case we have a function with two inputs, so it is not possible to define alternation properly over the two-dimensional domain. Our solution to this problem is addressed in Section 4. 
After $\left\{\mathrm{P}_{\mathrm{j}}\right\}_{1}^{\mathrm{n}}$ are found, the construction parameters are determined from $\mathrm{P}_{\mathrm{j}}$. If the number of coefficients $P_{j}=n$ is greater than the number of construction parameters, it means that the coefficients $P_{j}$ are interrelated. For illustration, consider the case where there are $n-2$ construction parameters, but $\left\{\mathrm{P}_{\mathrm{j}}\right\}_{1}^{\mathrm{n}}$. Let $P_{n-1}=\lambda_{1}, P_{n}=\lambda_{2}, P_{j}=\ell_{j}+m_{j} \lambda_{1}+n_{j} \lambda_{2}$ for $j=1, \ldots, n-2$ and $L=\ell+m \lambda_{1}+n \lambda_{2}$. Eq. (1) becomes

$$
\sum_{\mathrm{j}=1}^{\mathrm{n}-2}\left(\ell_{\mathrm{j}}+\mathrm{m}_{\mathrm{j}} \lambda_{1}+\mathrm{n}_{\mathrm{j}} \lambda_{2}\right) \mathrm{f}_{\mathrm{j}}\left(\mathbf{x}_{\mathrm{i}}\right)+\lambda_{1} \mathrm{f}_{\mathrm{n}-1}\left(\mathbf{x}_{\mathrm{i}}\right)+\lambda_{2} \mathrm{f}_{\mathrm{n}}\left(\mathbf{x}_{\mathrm{i}}\right)-\mathrm{F}\left(\mathbf{x}_{\mathbf{i}}\right)= \pm\left(\ell+\mathrm{m} \lambda_{1}+\mathrm{n} \lambda_{2}\right) \text { for } \mathrm{i}=1, \ldots, \mathrm{n}-1
$$

Equating the coefficients of $\lambda_{1}, \lambda_{2}$ and the remaining parts in Eq. (2) we obtain

$$
\sum_{j=1}^{n-2} \ell_{j} f_{j}\left(\mathbf{x}_{i}\right) \pm \ell=F\left(\mathbf{x}_{i}\right), \sum_{j=1}^{n-2} m_{j} f_{j}\left(\mathbf{x}_{i}\right) \pm m=-f_{n-1}\left(\mathbf{x}_{i}\right) \text { and } \sum_{j=1}^{n-2} n_{j} f_{j}\left(\mathbf{x}_{i}\right) \pm n=-f_{n}\left(\mathbf{x}_{i}\right), i=1, \ldots, n-1
$$

$\left\{\ell_{\mathrm{j}}\right\}_{1}^{\mathrm{n}-2},\left\{\mathrm{~m}_{\mathrm{j}}\right\}_{1}^{\mathrm{n}-2},\left\{\mathrm{n}_{\mathrm{j}}\right\}_{1}^{\mathrm{n}-2}, \ell, \mathrm{m}$ and $\mathrm{n}$ are solved linearly from Eq. (3). After the unknowns are determined, since there is a relationship between $\mathrm{P}_{\mathrm{n}-1}=\lambda_{1}, \mathrm{P}_{\mathrm{n}}=\lambda_{2}$ and some other coefficients $P_{j}=\ell_{j}+m_{j} \lambda_{1}+n_{j} \lambda_{2}, \lambda$ is solved from this relationship. $P_{j}=\ell_{j}+m_{j} \lambda_{1}+n_{j} \lambda_{2}$ for $j=1, \ldots, n-2$ and $\mathrm{L}=\ell+\mathrm{m} \lambda_{1}+\mathrm{n} \lambda_{2}$ are determined and the $\mathrm{n}-2$ construction parameters are solved from $\left\{\mathrm{P}_{\mathrm{j}}\right\}_{1}^{\mathrm{n}-1}$.

\section{I/O Equation and Error Analysis}

In this section we derive the I/O relationship of the mechanism to be used in Chebyshev approximation.

\subsection{Derivation of the I/O Equation for Spherical 5R Mechanism}

The kinematic representation and the parameters of five-bar mechanism are presented on the kinematic diagram shown in Fig. 1. A Cartesian reference frame $\mathrm{O}-\mathrm{xyz}$ is located at the center of the spherical mechanism such that the $\mathrm{z}$-axis points along one of the fixed joint axis and the two fixed joint axes define the xz-plane. Since the scale of the mechanism does not affect the I/O relationship, we assume that the radius of spherical surface equals to 1 . The construction parameters are $\left\{\alpha_{j}\right\}_{1}^{5}$, the input angles are $\theta$ and $\phi$ and the output angle is $\psi$ whereas the function to be synthesized is given by $\psi=\mathrm{f}(\theta, \phi)$.

The input and output joints are chosen as joints A, C, E; however, if the designer has the freedom to choose the input and output joints, the necessary formulation must be obtained for different configurations. For the case of a spherical five-bar mechanism, there are two alternative choices for the selection of these three joints: either all joints are adjacent to each other or one of the joints is separated from the other two which are adjacent. Considering the I/O relationship of the mechanism, which of these three joints is the output does not quite matter. Furthermore, it does not matter which joints will be the fixed joints because one can apply kinematic inversion before and after the synthesis. For example, selecting A, C, and E joint in Fig. 1 is equivalent to selecting A, B, and D. As it will be apparent from the formulations below, when the input and output joints are chosen such that one of the joints is separated from the other two, we obtain a linear set of equations. For conciseness, we do not provide the formulation for the case where the three joints are selected such that all three are adjacent to each other, but it can be shown that in that case the problem is highly nonlinear and an analytical solution is not possible. 
The I/O equation for the spherical $5 \mathrm{R}$ mechanism is determined by expressing the joint locations in terms of the inputs and the output and by making use of a simplification of the $5 \mathrm{R}$ mechanism to a $4 \mathrm{R}$ mechanism with a variable-length imaginary link represented by $\alpha_{6}$ as a function of the second input $\phi$ as depicted in Fig. 1.

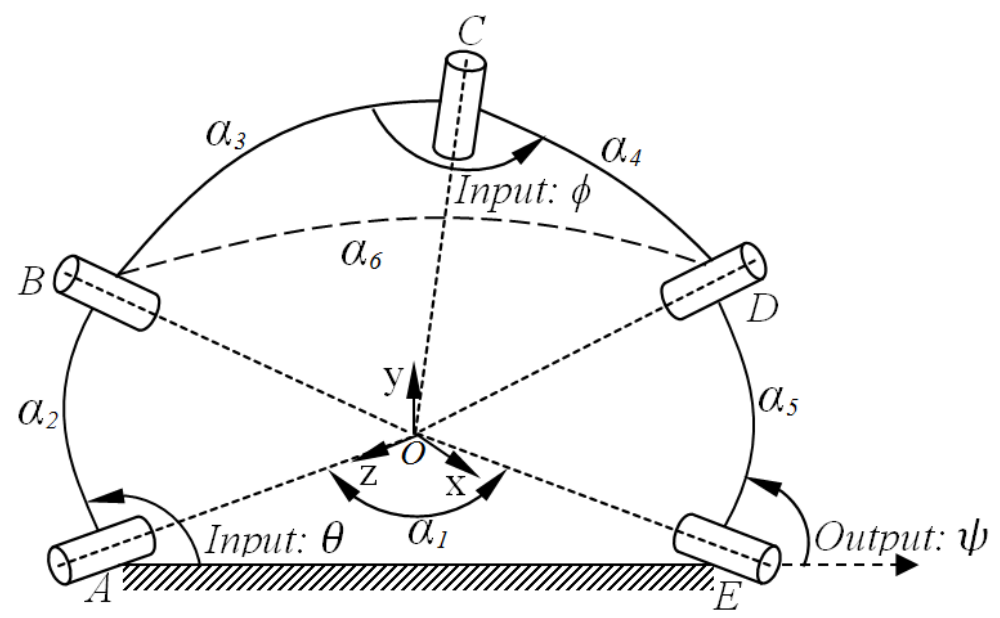

Fig. 1 Spherical five-bar mechanism

The coordinates of points $B$ and $D$ (see Figure 1) with respect to reference frame O-xyz can be described as follows:

$$
\overline{\mathrm{B}}=\mathrm{Z}(\theta) \mathrm{Y}\left(\alpha_{2}\right)\left[\begin{array}{l}
0 \\
0 \\
1
\end{array}\right]=\left[\begin{array}{c}
\mathrm{S} \alpha_{2} \mathrm{C} \theta \\
\mathrm{S} \alpha_{2} \mathrm{~S} \theta \\
\mathrm{C} \alpha_{2}
\end{array}\right] \quad \overline{\mathrm{D}}=\mathrm{Y}\left(\alpha_{1}\right) \mathrm{Z}(\psi) \mathrm{Y}\left(\alpha_{5}\right)\left[\begin{array}{l}
0 \\
0 \\
1
\end{array}\right]=\left[\begin{array}{c}
\mathrm{C} \psi \mathrm{S} \alpha_{5} \mathrm{C} \alpha_{1}+\mathrm{S} \alpha_{1} \mathrm{C} \alpha_{5} \\
\mathrm{~S} \psi \mathrm{S} \alpha_{5} \\
-\mathrm{C} \psi \mathrm{S} \alpha_{5} \mathrm{~S} \alpha_{1}+\mathrm{C} \alpha_{5} \mathrm{C} \alpha_{1}
\end{array}\right]
$$

where $\mathrm{Z}($.) and $\mathrm{Y}($.) are rotation matrices about $\mathrm{z}$ and y axes, respectively [34] and $\mathrm{S}$ and $\mathrm{C}$ represent the sine and cosine functions, respectively.

The scalar product of $\overline{\mathrm{B}}$ and $\overline{\mathrm{D}}$ vectors yields:

$$
\overline{\mathrm{B}} \cdot \overline{\mathrm{D}}=|\overline{\mathrm{B}}||\overline{\mathrm{D}}| \mathrm{C} \alpha_{6}
$$

Using spherical law of cosines for the spherical triangle BCD:

$$
\mathrm{C} \alpha_{3} \mathrm{C}_{4}+\mathrm{S}_{3} \mathrm{~S} \alpha_{4} \mathrm{C} \phi=\mathrm{C} \alpha_{6}
$$

Combining Eqs. (4), (5) and (6) we obtain the I/O equation of the spherical 5R mechanism:

$$
\frac{\mathrm{C} \alpha_{5} \mathrm{C} \alpha_{1} \mathrm{C} \alpha_{2}-\mathrm{C} \alpha_{3} \mathrm{C} \alpha_{4}}{\mathrm{~S} \alpha_{1} \mathrm{C} \alpha_{2} \mathrm{~S} \alpha_{5}}-\frac{\mathrm{S} \alpha_{3} \mathrm{~S} \alpha_{4}}{\mathrm{~S} \alpha_{1} \mathrm{C} \alpha_{2} \mathrm{~S} \alpha_{5}} \mathrm{C} \phi+\frac{\mathrm{T} \alpha_{2}}{\mathrm{~T} \alpha_{5}} \mathrm{C} \theta+\frac{\mathrm{T} \alpha_{2}}{\mathrm{~S} \alpha_{1}} \mathrm{~S} \psi \mathrm{S} \theta+\frac{\mathrm{T} \alpha_{2}}{\mathrm{~T} \alpha_{1}} \mathrm{C} \psi \mathrm{C} \theta-\mathrm{C} \psi=0
$$

where $\mathrm{T}$ represents the tangent function. The $\mathrm{I} / \mathrm{O}$ equation can be expressed in polynomial form:

$$
\sum_{j=1}^{5} P_{j} f_{j}\left(\mathbf{x}_{i}\right)-F\left(\mathbf{x}_{\mathbf{i}}\right)= \pm L \text { for } \mathrm{i}=1, \ldots, 6
$$

where $\mathbf{x}_{\mathrm{i}}$ represents the vector of the inputs $\theta_{\mathrm{i}}, \phi_{\mathrm{i}}$ and the output $\psi_{\mathrm{i}}, \mathrm{L}$ is the Chebyshev error and $\left\{\mathrm{P}_{\mathrm{j}}\right\}_{1}^{5}$ are the unknown coefficients defined by $\left\{\alpha_{j}\right\}_{1}^{5}$ as: 


$$
\mathrm{P}_{1}=\frac{\mathrm{C} \alpha_{5} \mathrm{C} \alpha_{1} \mathrm{C} \alpha_{2}-\mathrm{C} \alpha_{3} \mathrm{C} \alpha_{4}}{\mathrm{~S} \alpha_{1} \mathrm{C} \alpha_{2} \mathrm{~S} \alpha_{5}}, \mathrm{P}_{2}=\frac{-\mathrm{S} \alpha_{3} \mathrm{~S} \alpha_{4}}{\mathrm{~S} \alpha_{1} \mathrm{C} \alpha_{2} \mathrm{~S} \alpha_{5}}, \mathrm{P}_{3}=\frac{T \alpha_{2}}{\mathrm{~T} \alpha_{5}}, \mathrm{P}_{4}=\frac{\mathrm{T} \alpha_{2}}{\mathrm{~S} \alpha_{1}}, \mathrm{P}_{5}=\frac{\mathrm{T} \alpha_{2}}{\mathrm{~T} \alpha_{1}}
$$

and $\left\{\mathrm{f}_{\mathrm{j}}(\mathbf{x})\right\}_{1}^{5}$ and $\mathrm{F}(\mathbf{x})$ are functions of the design points $\theta_{\mathrm{i}}, \phi_{\mathrm{i}}$ and $\psi_{\mathrm{i}}$ where,

$$
\mathrm{f}_{1}\left(\mathbf{x}_{\mathbf{i}}\right)=1, \mathrm{f}_{2}\left(\mathbf{x}_{\mathbf{i}}\right)=\mathrm{C} \phi_{\mathrm{i}}, \mathrm{f}_{3}\left(\mathbf{x}_{\mathbf{i}}\right)=\mathrm{C} \theta_{\mathrm{i}}, \mathrm{f}_{4}\left(\mathbf{x}_{\mathbf{i}}\right)=\mathrm{S} \psi_{\mathrm{i}} \mathrm{S} \theta_{\mathrm{i}}, \mathrm{f}_{5}\left(\mathbf{x}_{\mathbf{i}}\right)=\mathrm{C} \psi_{\mathrm{i}} \mathrm{C} \theta_{\mathrm{i}} \text { and } \mathrm{F}\left(\mathbf{x}_{\mathbf{i}}\right)=\mathrm{C} \psi_{\mathrm{i}}
$$

Eq. (8) with five unknown parameters $\left\{\mathrm{P}_{\mathrm{j}}\right\}_{1}^{5}$ and the error parameter L requires six design points over the two-dimensional input domain. Eq. (8) can be written in matrix form as

$$
\left[\begin{array}{llllll}
\mathrm{f}_{1}\left(\mathbf{x}_{1}\right) & \mathrm{f}_{2}\left(\mathbf{x}_{1}\right) & \mathrm{f}_{3}\left(\mathbf{x}_{1}\right) & \mathrm{f}_{4}\left(\mathbf{x}_{1}\right) & \mathrm{f}_{5}\left(\mathbf{x}_{1}\right) & \pm 1 \\
\mathrm{f}_{1}\left(\mathbf{x}_{2}\right) & \mathrm{f}_{2}\left(\mathbf{x}_{2}\right) & \mathrm{f}_{3}\left(\mathbf{x}_{2}\right) & \mathrm{f}_{4}\left(\mathbf{x}_{2}\right) & \mathrm{f}_{5}\left(\mathbf{x}_{2}\right) & \pm 1 \\
\mathrm{f}_{1}\left(\mathbf{x}_{3}\right) & \mathrm{f}_{2}\left(\mathbf{x}_{3}\right) & \mathrm{f}_{3}\left(\mathbf{x}_{3}\right) & \mathrm{f}_{4}\left(\mathbf{x}_{3}\right) & \mathrm{f}_{5}\left(\mathbf{x}_{3}\right) & \pm 1 \\
\mathrm{f}_{1}\left(\mathbf{x}_{4}\right) & \mathrm{f}_{2}\left(\mathbf{x}_{4}\right) & \mathrm{f}_{3}\left(\mathbf{x}_{4}\right) & \mathrm{f}_{4}\left(\mathbf{x}_{4}\right) & \mathrm{f}_{5}\left(\mathbf{x}_{4}\right) & \pm 1 \\
\mathrm{f}_{1}\left(\mathbf{x}_{5}\right) & \mathrm{f}_{2}\left(\mathbf{x}_{5}\right) & \mathrm{f}_{3}\left(\mathbf{x}_{5}\right) & \mathrm{f}_{4}\left(\mathbf{x}_{5}\right) & \mathrm{f}_{5}\left(\mathbf{x}_{5}\right) & \pm 1 \\
\mathrm{f}_{1}\left(\mathbf{x}_{6}\right) & \mathrm{f}_{2}\left(\mathbf{x}_{6}\right) & \mathrm{f}_{3}\left(\mathbf{x}_{6}\right) & \mathrm{f}_{4}\left(\mathbf{x}_{6}\right) & \mathrm{f}_{5}\left(\mathbf{x}_{6}\right) & \pm 1
\end{array}\right]\left[\begin{array}{l}
\mathrm{F}\left(\mathbf{x}_{1}\right) \\
\mathrm{P}_{2} \\
\mathrm{P}_{3} \\
\mathrm{P}_{4} \\
\left.\mathrm{P}_{5}\right) \\
\mathrm{L}\left(\mathbf{x}_{3}\right) \\
\mathrm{F}\left(\mathbf{x}_{4}\right) \\
\mathrm{F}\left(\mathbf{x}_{5}\right) \\
\mathrm{F}\left(\mathbf{x}_{6}\right)
\end{array}\right]
$$

Thus, unknown parameters $\left\{\mathrm{P}_{\mathrm{j}}\right\}_{1}^{5}$ and the error $\mathrm{L}$ can be solved uniquely for the function generation synthesis problem with Chebyshev approximation. In Eq. (10), the selection of signs in the last column of the coefficient matrix changes during the iterations, as illustrated in Section 4.

After sufficient number of iterations $\left\{\mathrm{P}_{\mathrm{j}}\right\}_{1}^{5}$ are determined and the construction parameters are solved from Eq. (9) as follows:

$$
\alpha_{1}= \pm \operatorname{Cos}^{-1} \frac{P_{5}}{P_{4}}, \alpha_{2}=\operatorname{Tan}^{-1}\left(P_{4} S \alpha_{1}\right), \alpha_{5}=\operatorname{Tan}^{-1} \frac{T \alpha_{2}}{P_{3}}, \alpha_{3}=\frac{E_{1} \pm E_{2}}{2}, \alpha_{4}=\frac{E_{1} \mp E_{2}}{2}
$$

where $\mathrm{E}_{1}=\operatorname{Cos}^{-1}\left[\mathrm{C} \alpha_{2}\left(\mathrm{C} \alpha_{1} \mathrm{C} \alpha_{5}-\left(\mathrm{P}_{1}+\mathrm{P}_{2}\right) \mathrm{S} \alpha_{1} \mathrm{~S} \alpha_{5}\right)\right], \mathrm{E}_{2}=\operatorname{Cos}^{-1}\left[\mathrm{C} \alpha_{2}\left(\mathrm{C} \alpha_{1} \mathrm{C} \alpha_{5}-\left(\mathrm{P}_{1}-\mathrm{P}_{2}\right) \mathrm{S} \alpha_{1} \mathrm{~S} \alpha_{5}\right)\right]$.

\subsection{Derivation of I/O Equations with Initial Positions of Input-Output Angles $(\theta, \phi, \psi)$}

Extra construction parameters can be introduced when we change the reference with respect to which the input-output angles are measured. When there are more construction parameters, we can use more design points and increase the accuracy. Instead of $\theta, \phi$ and $\psi$ we use in Section 3.1, we may use $\theta=\theta_{0}$ $+\theta_{\mathrm{p}}, \phi=\phi_{0}+\phi_{\mathrm{p}}$ and $\psi=\psi_{0}+\psi_{\mathrm{p}}$ such that $\theta_{0}, \phi_{0}$ and $\psi_{0}$ are the initial constant angles to be designed and $\theta_{\mathrm{p}}, \phi_{\mathrm{p}}$ and $\psi_{\mathrm{p}}$ are the new joint variables.

- Firstly, let us introduce $\phi=\phi_{0}+\phi_{\mathrm{p}}$ into Eq. (7):

$$
\begin{gathered}
\frac{\mathrm{C} \alpha_{5} \mathrm{C} \alpha_{1} \mathrm{C} \alpha_{2}-\mathrm{C} \alpha_{3} \mathrm{C} \alpha_{4}}{\mathrm{~S} \alpha_{1} \mathrm{C} \alpha_{2} \mathrm{~S} \alpha_{5}}-\frac{\mathrm{S} \alpha_{3} \mathrm{~S} \alpha_{4} \mathrm{C} \phi_{0}}{\mathrm{~S} \alpha_{1} \mathrm{C} \alpha_{2} \mathrm{~S} \alpha_{5}} \mathrm{C} \phi_{\mathrm{p}}+\frac{\mathrm{S} \alpha_{3} \mathrm{~S} \alpha_{4} \mathrm{~S} \phi_{0}}{\mathrm{~S} \alpha_{1} \mathrm{C} \alpha_{2} \mathrm{~S} \alpha_{5}} \mathrm{~S} \phi_{\mathrm{p}}+\frac{\mathrm{T} \alpha_{2}}{\mathrm{~T} \alpha_{5}} \mathrm{C} \theta \\
+\frac{\mathrm{T} \alpha_{2}}{\mathrm{~S} \alpha_{1}} \mathrm{~S} \psi \mathrm{S} \theta+\frac{\mathrm{T} \alpha_{2}}{\mathrm{~T} \alpha_{1}} \mathrm{C} \psi \mathrm{C} \theta-\mathrm{C} \psi=0
\end{gathered}
$$

Representation of the I/O equation in polynomial form can be defined as: 


$$
\sum_{j=1}^{6} P_{j} f_{j}\left(\mathbf{x}_{i}\right)-F\left(\mathbf{x}_{\mathbf{i}}\right)= \pm L \text { for } \mathrm{i}=1, \ldots, 7
$$

where, $\left\{\mathrm{P}_{\mathrm{j}}\right\}_{1}^{6}$ are:

$$
\mathrm{P}_{1}=\frac{\mathrm{C} \alpha_{5} \mathrm{C} \alpha_{1} \mathrm{C} \alpha_{2}-\mathrm{C} \alpha_{3} \mathrm{C} \alpha_{4}}{\mathrm{~S} \alpha_{1} \mathrm{C} \alpha_{2} \mathrm{~S} \alpha_{5}}, \mathrm{P}_{2}=-\frac{\mathrm{S} \alpha_{3} \mathrm{~S} \alpha_{4} \mathrm{C} \phi_{0}}{\mathrm{~S} \alpha_{1} \mathrm{C} \alpha_{2} \mathrm{~S} \alpha_{5}}, \mathrm{P}_{3}=\frac{\mathrm{S} \alpha_{3} \mathrm{~S} \alpha_{4} \mathrm{~S} \phi_{0}}{\mathrm{~S} \alpha_{1} \mathrm{C} \alpha_{2} \mathrm{~S} \alpha_{5}}, \mathrm{P}_{4}=\frac{\mathrm{T} \alpha_{2}}{\mathrm{~T} \alpha_{5}}, \mathrm{P}_{5}=\frac{\mathrm{T} \alpha_{2}}{\mathrm{~S} \alpha_{1}}, \mathrm{P}_{6}=\frac{\mathrm{T} \alpha_{2}}{\mathrm{~T} \alpha_{1}}
$$

and $\left\{f_{j}(\mathbf{x})\right\}_{1}^{6}, F_{j}(\mathbf{x})$ are the functions

$$
\mathrm{f}_{1}\left(\mathbf{x}_{\mathbf{i}}\right)=1, \mathrm{f}_{2}\left(\mathbf{x}_{\mathbf{i}}\right)=\mathrm{C} \phi_{\mathrm{pi}}, \mathrm{f}_{3}\left(\mathbf{x}_{\mathbf{i}}\right)=\mathrm{S} \phi_{\mathrm{pi}}, \mathrm{f}_{4}\left(\mathbf{x}_{\mathbf{i}}\right)=\mathrm{C} \theta_{\mathrm{i}}, \mathrm{f}_{5}\left(\mathbf{x}_{\mathbf{i}}\right)=\mathrm{S} \psi_{\mathrm{i}} \mathrm{S} \theta_{\mathrm{i}}, \mathrm{f}_{6}\left(\mathbf{x}_{\mathbf{i}}\right)=\mathrm{C} \psi_{\mathrm{i}} \mathrm{C} \theta_{\mathrm{i}} \text { and } \mathrm{F}\left(\mathbf{x}_{\mathbf{i}}\right)=\mathrm{C} \psi_{\mathrm{i}}
$$

Given seven design points, $\left\{\mathrm{P}_{\mathrm{j}}\right\}_{1}^{6}$ and $\mathrm{L}$ can be solved linearly from Eq. (12). Then, the construction parameters are solved from Eq. (13) as

$$
\phi_{0}=\operatorname{Tan}^{-1}\left(-\frac{\mathrm{P}_{3}}{\mathrm{P}_{2}}\right), \alpha_{1}=\operatorname{Cos}^{-1} \frac{\mathrm{P}_{6}}{\mathrm{P}_{5}}, \alpha_{2}=\operatorname{Tan}^{-1}\left(\mathrm{P}_{5} \mathrm{~S} \alpha_{1}\right), \alpha_{5}=\operatorname{Tan}^{-1} \frac{\operatorname{T\alpha }_{2}}{\mathrm{P}_{4}}, \alpha_{3}=\frac{\mathrm{E}_{1} \pm \mathrm{E}_{2}}{2}, \alpha_{4}=\frac{\mathrm{E}_{1} \mp \mathrm{E}_{2}}{2}
$$

where $\mathrm{E}_{1}=\mathrm{Cos}^{-1}\left[\mathrm{C} \alpha_{2}\left(\mathrm{C} \alpha_{1} \mathrm{C} \alpha_{5}-\left(\mathrm{P}_{1}+\frac{\mathrm{P}_{2}}{\mathrm{C} \phi_{0}}\right) \mathrm{S} \alpha_{1} \mathrm{~S} \alpha_{5}\right)\right]$,

$\mathrm{E}_{2}=\mathrm{Cos}^{-1}\left[\mathrm{C} \alpha_{2}\left(\mathrm{C} \alpha_{1} \mathrm{C} \alpha_{5}-\left(\mathrm{P}_{1}-\frac{\mathrm{P}_{2}}{\mathrm{C} \phi_{0}}\right) \mathrm{S} \alpha_{1} \mathrm{~S} \alpha_{5}\right)\right]$.

- Similar with the other input angle, applying newly defined $\theta$ as $\theta=\theta_{0}+\theta_{\mathrm{p}}$, where $\theta_{0}$ is the constant angle measured from the xz-plane about the z-axis in Fig. 1 and $\theta_{\mathrm{p}}$ is the variable input angle measured further from $\theta_{0}$. Modifying Eq. (7) for $\theta=\theta_{0}+\theta_{\mathrm{p}}$ :

$$
\begin{gathered}
\frac{\mathrm{C} \alpha_{1} \mathrm{C} \alpha_{2} \mathrm{C} \alpha_{5}-\mathrm{C} \alpha_{3} \mathrm{C} \alpha_{4}}{\mathrm{~S} \alpha_{2} \mathrm{~S} \alpha_{5} \mathrm{C} \theta_{0}}-\frac{\mathrm{S} \alpha_{3} \mathrm{~S} \alpha_{4}}{\mathrm{~S} \alpha_{2} \mathrm{~S} \alpha_{5} \mathrm{C} \theta_{0}} \mathrm{C} \phi-\frac{\mathrm{S} \alpha_{1}}{\mathrm{~T} \alpha_{2} \mathrm{C} \theta_{0}} \mathrm{C} \psi+\mathrm{C} \alpha_{1} \mathrm{C} \psi \mathrm{C} \theta_{\mathrm{p}}+\mathrm{T} \theta_{0} \mathrm{~S} \psi \mathrm{C} \theta_{\mathrm{p}} \\
+\frac{\mathrm{S} \alpha_{1}}{\mathrm{~T} \alpha_{5}} \mathrm{C} \theta_{\mathrm{p}}-\frac{\mathrm{S} \alpha_{1} \mathrm{~T} \theta_{0}}{\mathrm{~T} \alpha_{5}} \mathrm{~S} \theta_{\mathrm{p}}-\mathrm{C} \alpha_{1} \mathrm{~T} \theta_{0} \mathrm{C} \psi \mathrm{S} \theta_{\mathrm{p}}+\mathrm{S} \psi \mathrm{S} \theta_{\mathrm{p}}=0
\end{gathered}
$$

The I/O equation can be expressed in polynomial form as

$$
\sum_{\mathrm{j}=1}^{8} \mathrm{P}_{\mathrm{j}} \mathrm{f}_{\mathrm{j}}\left(\mathbf{x}_{\mathbf{i}}\right)-\mathrm{F}\left(\mathbf{x}_{\mathbf{i}}\right)= \pm \mathrm{L} \text { for } \mathrm{i}=1, \ldots, 7
$$

where the unknown coefficients $\left\{\mathrm{P}_{\mathrm{j}}\right\}_{1}^{8}$ are

$$
\begin{gathered}
\mathrm{P}_{1}=\frac{\mathrm{C} \alpha_{1} \mathrm{C} \alpha_{2} \mathrm{C} \alpha_{5}-\mathrm{C} \alpha_{3} \mathrm{C} \alpha_{4}}{\mathrm{~S} \alpha_{2} \mathrm{~S} \alpha_{5} \mathrm{C} \theta_{0}}, \mathrm{P}_{2}=-\frac{\mathrm{S} \alpha_{3} \mathrm{~S} \alpha_{4}}{\mathrm{~S} \alpha_{2} \mathrm{~S} \alpha_{5} \mathrm{C} \theta_{0}}, \mathrm{P}_{3}=-\frac{\mathrm{S} \alpha_{1}}{\mathrm{~T} \alpha_{2} \mathrm{C} \theta_{0}}, \mathrm{P}_{4}=\mathrm{C} \alpha_{1}, \mathrm{P}_{5}=\mathrm{T} \theta_{0}, \\
\mathrm{P}_{6}=\frac{\mathrm{S} \alpha_{1}}{\mathrm{~T} \alpha_{5}}, \mathrm{P}_{7}=\frac{\mathrm{S} \alpha_{1} \mathrm{~T} \theta_{0}}{\mathrm{~T} \alpha_{5}}, \mathrm{P}_{8}=-\mathrm{C} \alpha_{1} \mathrm{~T} \theta_{0}
\end{gathered}
$$

and $\left\{\mathrm{f}_{\mathrm{j}}(\mathbf{x})\right\}_{1}^{8}, \mathrm{~F}_{\mathrm{j}}(\mathbf{x})$ are 


$$
\begin{gathered}
\mathrm{f}_{1}\left(\mathbf{x}_{\mathbf{i}}\right)=1, \mathrm{f}_{2}\left(\mathbf{x}_{\mathbf{i}}\right)=\mathrm{C} \phi, \mathrm{f}_{3}\left(\mathbf{x}_{\mathbf{i}}\right)=\mathrm{C} \psi, \mathrm{f}_{4}\left(\mathbf{x}_{\mathbf{i}}\right)=\mathrm{C} \psi \mathrm{C} \theta_{\mathrm{p}}, \mathrm{f}_{5}\left(\mathbf{x}_{\mathbf{i}}\right)=\mathrm{S} \psi \mathrm{C} \theta_{\mathrm{p}}, \\
\mathrm{f}_{6}\left(\mathbf{x}_{\mathbf{i}}\right)=\mathrm{C} \theta_{\mathrm{p}}, \mathrm{f}_{7}\left(\mathbf{x}_{\mathbf{i}}\right)=-\mathrm{S} \theta_{\mathrm{p}}, \mathrm{f}_{8}\left(\mathbf{x}_{\mathbf{i}}\right)=\mathrm{C} \psi \mathrm{S} \theta_{\mathrm{p}} \text { and } \mathrm{F}\left(\mathbf{x}_{\mathbf{i}}\right)=-\mathrm{S} \psi \mathrm{S} \theta_{\mathrm{p}}
\end{gathered}
$$

There are eight coefficients $\mathrm{P}_{\mathrm{j}}$, but six construction parameters. Two of the $\mathrm{P}_{\mathrm{j}}$ depend on the others as follows:

$$
\mathrm{P}_{7}=\mathrm{P}_{5} \mathrm{P}_{6} \text { and } \mathrm{P}_{8}=-\mathrm{P}_{4} \mathrm{P}_{5}
$$

Let $\mathrm{P}_{7}=\lambda_{1}$ and $\mathrm{P}_{8}=\lambda_{2}, \mathrm{P}_{\mathrm{j}}=\ell_{\mathrm{j}}+\mathrm{m}_{\mathrm{j}} \lambda_{1}+\mathrm{n}_{\mathrm{j}} \lambda_{2}$ for $\mathrm{j}=1, \ldots, 6$ and $\mathrm{L}=\ell+\mathrm{m} \lambda_{1}+\mathrm{n} \lambda_{2}$. When we apply the linear solution procedure explained in Section $2, \ell_{j}, m_{j}, n_{j}, \ell, m$ and $n$ are determined uniquely. Next, $\lambda_{1}$ and $\lambda_{2}$ should be solved from Eq. (16):

$$
\left(\ell_{5}+\mathrm{m}_{5} \lambda_{1}+\mathrm{n}_{5} \lambda_{2}\right)\left(\ell_{6}+\mathrm{m}_{6} \lambda_{1}+\mathrm{n}_{6} \lambda_{2}\right)-\lambda_{1}=0 \text { and }\left(\ell_{4}+\mathrm{m}_{4} \lambda_{1}+\mathrm{n}_{4} \lambda_{2}\right)\left(\ell_{5}+\mathrm{m}_{5} \lambda_{1}+\mathrm{n}_{5} \lambda_{2}\right)+\lambda_{2}=0(17
$$

Eliminating either of $\lambda_{1}$ or $\lambda_{2}$ form Eq. (17) results in a cubic equation in the remaining variable. The details of this elimination can be seen in [26]. The cubic equation can be solved analytically and there is either single real solution, or three real solutions. In case of three real solutions, the designer shall use the solution which yields less error. Once $\lambda_{1}$ and $\lambda_{2}$ are determined, $P_{j}=\ell_{j}+m_{j} \lambda_{1}+n_{j} \lambda_{2}$ for $j=$ $1, \ldots, 6$ and the construction parameters are solved from $\left\{\mathrm{P}_{\mathrm{j}}\right\}_{1}^{6}$ as

$$
\theta_{0}=\operatorname{Tan}^{-1} P_{5}, \alpha_{1}=\operatorname{Cos}^{-1} P_{4}, \alpha_{2}=\operatorname{Tan}^{-1}\left(-\frac{S \alpha_{1}}{P_{3} \theta_{0}}\right), \alpha_{5}=\operatorname{Tan}^{-1} \frac{S \alpha_{1}}{P_{6}}, \alpha_{3}=\frac{E_{1} \pm E_{2}}{2}, \alpha_{4}=\frac{E_{1} \mp E_{2}}{2}
$$

where $\mathrm{E}_{1}=\mathrm{Cos}^{-1}\left(\mathrm{C} \alpha_{1} \mathrm{C} \alpha_{2} \mathrm{C} \alpha_{5}-\left(\mathrm{P}_{1}+\mathrm{P}_{2}\right) \mathrm{S} \alpha_{2} \mathrm{~S} \alpha_{5} \mathrm{C} \theta_{0}\right)$,

$$
\mathrm{E}_{2}=\mathrm{Cos}^{-1}\left(\mathrm{C} \alpha_{1} \mathrm{C \alpha}_{2} \mathrm{C} \alpha_{5}-\left(\mathrm{P}_{1}-\mathrm{P}_{2}\right) \mathrm{S} \alpha_{2} \mathrm{~S}_{5} \mathrm{C} \theta_{0}\right) \text {. }
$$

- Finally, substituting $\psi=\psi_{0}+\psi_{\mathrm{p}}$ into Eq. (7):

$$
\begin{gathered}
\frac{\mathrm{C} \alpha_{1} \mathrm{C} \alpha_{2} \mathrm{C} \alpha_{5}-\mathrm{C} \alpha_{3} \mathrm{C} \alpha_{4}}{\mathrm{~S} \alpha_{1} \mathrm{C} \alpha_{2} \mathrm{~S} \alpha_{5} \mathrm{~S} \psi_{0}}-\frac{\mathrm{S} \alpha_{3} \mathrm{~S} \alpha_{4}}{\mathrm{~S} \alpha_{1} \mathrm{C} \alpha_{2} \mathrm{~S} \alpha_{5} \mathrm{~S} \psi_{0}} \mathrm{C} \phi+\frac{\mathrm{T} \alpha_{2}}{\mathrm{~T} \alpha_{5} \mathrm{~S} \psi_{0}} \mathrm{C} \theta-\frac{1}{\mathrm{~T} \psi_{0}} \mathrm{C} \psi_{\mathrm{p}}-\frac{\mathrm{T} \alpha_{2}}{\mathrm{~T} \alpha_{1}} \mathrm{C} \theta \mathrm{S} \psi_{\mathrm{p}} \\
+\frac{\mathrm{T} \alpha_{2}}{\mathrm{~S} \alpha_{1}} \mathrm{~S} \theta \mathrm{C} \psi_{\mathrm{p}}+\frac{\mathrm{T} \alpha_{2}}{\mathrm{~T} \alpha_{1} \mathrm{~T} \psi_{0}} \mathrm{C} \theta \mathrm{C} \psi_{\mathrm{p}}+\frac{\mathrm{T} \alpha_{2}}{\mathrm{~S} \alpha_{1} \mathrm{~T} \psi_{0}} \mathrm{~S} \theta \mathrm{S} \psi_{\mathrm{p}}+\mathrm{S} \psi_{\mathrm{p}}=0
\end{gathered}
$$

Eq. (18) can be expressed in polynomial form as Eq. (14), where $\left\{\mathrm{P}_{\mathrm{j}}\right\}_{1}^{8}$ are

$$
\begin{gathered}
\mathrm{P}_{1}=\frac{\mathrm{C} \alpha_{1} \mathrm{C} \alpha_{2} \mathrm{C} \alpha_{5}-\mathrm{C} \alpha_{3} \mathrm{C} \alpha_{4}}{\mathrm{~S} \alpha_{1} \mathrm{C} \alpha_{2} \mathrm{~S} \alpha_{5} \mathrm{~S} \psi_{0}}, \mathrm{P}_{2}=-\frac{\mathrm{S} \alpha_{3} \mathrm{~S} \alpha_{4}}{\mathrm{~S} \alpha_{1} \mathrm{C} \alpha_{2} \mathrm{~S} \alpha_{5} \mathrm{~S} \psi_{0}}, \mathrm{P}_{3}=\frac{\mathrm{T} \alpha_{2}}{\mathrm{~T} \alpha_{5} \mathrm{~S} \psi_{0}}, \mathrm{P}_{4}=-\frac{1}{\mathrm{~T} \psi_{0}}, \\
\mathrm{P}_{5}=-\frac{\mathrm{T} \alpha_{2}}{\mathrm{~T} \alpha_{1}}, \mathrm{P}_{6}=\frac{\mathrm{T} \alpha_{2}}{\mathrm{~S} \alpha_{1}}, \mathrm{P}_{7}=\frac{\mathrm{T} \alpha_{2}}{\mathrm{~T} \alpha_{1} \mathrm{~T} \psi_{0}}, \mathrm{P}_{8}=\frac{\mathrm{T} \alpha_{2}}{\mathrm{~S} \alpha_{1} \mathrm{~T} \psi_{0}}
\end{gathered}
$$

and $\left\{\mathrm{f}_{\mathrm{j}}(\mathbf{x})\right\}_{1}^{7}, \mathrm{~F}_{\mathrm{j}}(\mathbf{x})$ are

$$
\begin{gathered}
\mathrm{f}_{1}\left(\mathbf{x}_{\mathbf{i}}\right)=1, \mathrm{f}_{2}\left(\mathbf{x}_{\mathbf{i}}\right)=\mathrm{C} \phi, \mathrm{f}_{3}\left(\mathbf{x}_{\mathbf{i}}\right)=\mathrm{C} \theta, \mathrm{f}_{4}\left(\mathbf{x}_{\mathbf{i}}\right)=\mathrm{C} \psi_{\mathrm{p}}, \mathrm{f}_{5}\left(\mathbf{x}_{\mathbf{i}}\right)=\mathrm{C} \theta \mathrm{S} \psi_{\mathrm{p}}, \\
\mathrm{f}_{6}\left(\mathbf{x}_{\mathbf{i}}\right)=\mathrm{S} \theta \mathrm{C} \psi_{\mathrm{p}}, \mathrm{f}_{7}\left(\mathbf{x}_{\mathbf{i}}\right)=\mathrm{C} \theta \mathrm{C} \psi_{\mathrm{p}}, \mathrm{f}_{8}\left(\mathbf{x}_{\mathbf{i}}\right)=\mathrm{S} \theta \mathrm{S} \psi_{\mathrm{p}} \text { and } \mathrm{F}\left(\mathbf{x}_{\mathbf{i}}\right)=-\mathrm{S} \psi_{\mathrm{p}}
\end{gathered}
$$

In the polynomial representation of the spherical 5R mechanism, there are eight unknowns but only six linear equations. 


$$
\mathrm{P}_{7}=\mathrm{P}_{4} \mathrm{P}_{5} \text { and } \mathrm{P}_{8}=-\mathrm{P}_{4} \mathrm{P}_{6}
$$

Eq. (20) have the same form as in Eq. (16) and the solution procedure is as described above. The construction parameters of the mechanism are solved from Eq. (19) as follows:

$$
\psi_{0}=\operatorname{Tan}^{-1}\left(-\frac{1}{P_{4}}\right), \alpha_{1}=\operatorname{Cos}^{-1}\left(-\frac{P_{5}}{P_{6}}\right), \alpha_{2}=\operatorname{Tan}^{-1}\left(P_{6} S \alpha_{1}\right), \alpha_{5}=\operatorname{Tan}^{-1} \frac{T \alpha_{2}}{P_{3} S \psi_{0}}, \alpha_{3}=\frac{E_{1} \pm E_{2}}{2}, \alpha_{4}=\frac{E_{1} \mp E_{2}}{2}
$$

where $\mathrm{E}_{1}=\mathrm{Cos}^{-1}\left[\mathrm{C} \alpha_{2}\left(\mathrm{Ca}_{1} \mathrm{Ca}_{5}-\left(\mathrm{P}_{1}+\mathrm{P}_{2}\right) \mathrm{S} \alpha_{1} \mathrm{~S} \alpha_{5} \mathrm{~S} \psi_{0}\right)\right]$,

$$
\mathrm{E}_{2}=\mathrm{Cos}^{-1}\left[\mathrm{C} \alpha_{2}\left(\mathrm{C} \alpha_{1} \mathrm{C} \alpha_{5}-\left(\mathrm{P}_{1}-\mathrm{P}_{2}\right) \mathrm{S} \alpha_{1} \mathrm{~S} \alpha_{5} \mathrm{~S} \psi_{0}\right)\right] \text {. }
$$

\section{The Synthesis Problem}

Let the function to be generated be $\mathrm{z}=\mathrm{f}(\mathrm{x}, \mathrm{y})$ for $\mathrm{x}_{\min } \leq \mathrm{x} \leq \mathrm{x}_{\max }, \mathrm{y}_{\min } \leq \mathrm{y} \leq \mathrm{y}_{\max }$ and $\mathrm{z}_{\min } \leq \mathrm{z} \leq \mathrm{z}_{\max }$. The independent variables $x$ and $y$ should be related to the mechanism inputs $\theta$ and $\phi$ and the dependent variable $\mathrm{z}$ should be related to the mechanism output $\psi$. Depending on the application some or all of the function $z=f(x, y), x_{\min }, x_{\max }, y_{\min }$ and $y_{\max }$ may be demanded by the specific task. However, the designer can freely select the limits $\theta_{\min }, \theta_{\max }, \phi_{\min }, \phi_{\max }, \psi_{\min }$ and $\psi_{\max }$ of the mechanism inputs and output.

We shall linearly relate $\mathrm{x}$ to $\theta, \mathrm{y}$ to $\phi$ and $\mathrm{z}$ to $\psi$ as

$$
\frac{\mathrm{x}-\mathrm{x}_{\min }}{\mathrm{x}_{\max }-\mathrm{x}_{\min }}=\frac{\theta-\theta_{\min }}{\theta_{\max }-\theta_{\min }}, \frac{\mathrm{y}-\mathrm{y}_{\min }}{\mathrm{y}_{\max }-\mathrm{y}_{\min }}=\frac{\phi-\phi_{\min }}{\phi_{\max }-\phi_{\min }}, \frac{\mathrm{z}-\mathrm{z}_{\min }}{\mathrm{z}_{\max }-\mathrm{z}_{\min }}=\frac{\psi-\psi_{\min }}{\psi_{\max }-\psi_{\min }}
$$

Then desired $\psi, \theta$ and $\phi$ values for given $\mathrm{x}, \mathrm{y}, \mathrm{z}$ are found from Eq. (21) as follows:

$$
\theta=\frac{\theta_{\text {max }}-\theta_{\min }}{\mathrm{x}_{\max }-\mathrm{x}_{\min }}\left(\mathrm{x}-\mathrm{x}_{\min }\right)+\theta_{\min }, \phi=\frac{\phi_{\max }-\phi_{\min }}{\mathrm{y}_{\max }-\mathrm{y}_{\min }}\left(\mathrm{y}-\mathrm{y}_{\min }\right)+\phi_{\min }, \psi=\frac{\psi_{\max }-\psi_{\min }}{\mathrm{z}_{\max }-\mathrm{z}_{\min }}\left(\mathrm{z}-\mathrm{z}_{\min }\right)+\psi_{\min }
$$

and conversely

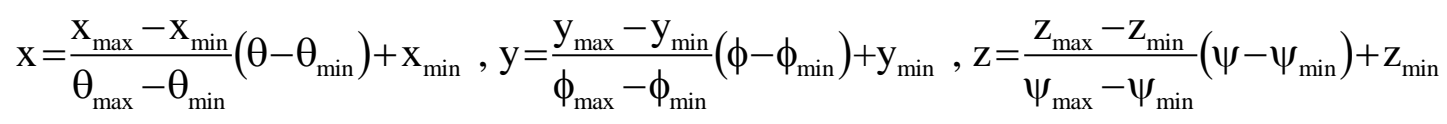

We use Eq. (22) for determining the design points $\left\{\theta_{\mathrm{i}}\right\}_{1}^{6},\left\{\phi_{\mathrm{i}}\right\}_{1}^{6}$ and $\left\{\psi_{\mathrm{i}}\right\}_{1}^{6}$ from $\left\{\mathrm{x}_{\mathrm{i}}\right\}_{1}^{6},\left\{\mathrm{y}_{\mathrm{i}}\right\}_{1}^{6}$ and $\left\{z_{i}\right\}_{1}^{6}=\left\{f\left(x_{i}, y_{i}\right)\right\}_{1}^{6}$. We use Eq. (23) after the synthesis is done and we need to check the error in between $\mathrm{Z}_{\text {desired }}=\mathrm{f}(\mathrm{x}, \mathrm{y})$ and $\mathrm{z}_{\text {generated }}$ with the mechanism. A good measure of this error is the percentage error defined as

$$
\% \text { Error }=100 \times \frac{\mathrm{z}_{\text {desired }}-\mathrm{z}_{\text {generated }}}{\mathrm{z}_{\text {desired }}}
$$

When evaluating $z_{g e n e r a t e d}$, one shall determine the output values of the mechanism for several given input values, say 100 values, by solving the I/O relationship.

It is necessary to select an initial spacing for the design points $\left\{\mathrm{x}_{\mathrm{i}}, \mathrm{y}_{\mathrm{i}}\right\}_{1}^{6}$ on the two dimensional domain defined by $\mathrm{x}_{\min } \leq \mathrm{x} \leq \mathrm{x}_{\max }, \mathrm{y}_{\min } \leq \mathrm{y} \leq \mathrm{y}_{\max }$ and also the limits of the sub-regions for iteration of each design point needs to be determined. We tried several alternative ways of spacing. In [25] we had five 
design points and selected four of the initial design points at the corners of the rectangular domain and the remaining one at the center. During the iterations, the points at the corners remained, while the other point was relocated at the location of maximum error at each iteration step. This type of spacing has the drawback that only one of the design points can be relocated and this limits the amount of enhancement in terms of error at each iteration step. In this study, we first tried to locate the initial position of four of the design points on the four respective sides and locate the other two inside the domain. We relocated the points on the boundaries along the respective sides according to the maximum errors and the points inside were relocated inside the domain. This modification still did not result in the sufficient mobility for the design points and the simulation results were not satisfactory. Finally we decided on a different type of spacing where four of the initial design points are in rectangular regions about the corners and the remaining two are in the remaining region, as depicted in Fig. 2. We call this spacing as regional spacing. Regional spacing allows the design points to be selected and relocated in larger domains. By this way, the design points have more freedom for relocation in between iteration steps and this increases the probability to obtain better results at each iteration step.

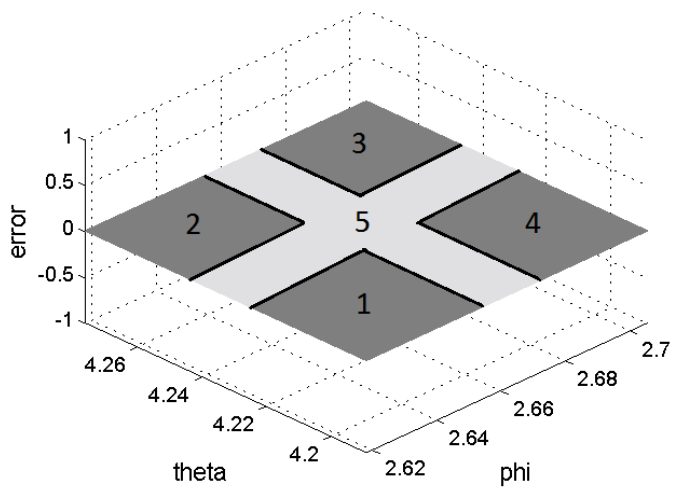

Fig. 2 Regional Spacing

Although this spacing method is superior to the other spacing methods mentioned, it still does not guarantee minimization of the maximum absolute error and may even diverge. Initially, the errors of the design points in regions 1-4 in Fig. 2 are equated to $+\mathrm{L}$ and the errors of the remaining two design points in region 5 are equated to $+\mathrm{L}$ for one and $-\mathrm{L}$ for the other. During the iterations, the design points in regions 1-4 are relocated to the points in their respective regions, where the extremum error value is attained, while the two design points in region 5 are relocated to the points where minimum and maximum errors are attained in that region. 


\section{Case Study with Regional Spacing for Six Design Points}

A case study is worked out using the I/O equation given by Eqs. $(7,8)$ for generation of the function $\mathrm{z}$ $=\mathrm{x}^{0.45} \mathrm{y}^{0.6}$ for $6 \leq \mathrm{x} \leq 10$ and $8 \leq \mathrm{y} \leq 12$. After several trials, the mechanism input/output angle limits are chosen as $60^{\circ} \leq \theta \leq 120^{\circ}, 80^{\circ} \leq \phi \leq 130^{\circ}$ and $75^{\circ} \leq \psi \leq 135^{\circ}$. The authors are aware of the fact that this is a purely academic example without any particular geometric meaning. The result of the optimization is sensitive against shifting the boundaries of the variables, and the periodic character of the angle measures is neglected.

The error variation plots and minimum and maximum error values at each iteration step are given in Table 1. The coloring in the figures in Table 1 range from blue (minimum) to red (maximum). After the first calculation, the maximum absolute errors are attained for $\left\{\theta_{i}\right\}_{1}^{6},\left\{\phi_{i}\right\}_{1}^{6}$ and the design points are modified accordingly. At each iteration step, the maximum and minimum approximation error are calculated and compared. The shape of the error surface stabilizes and the maximum and minimum errors reach close to their stable values at the fourth iteration, meaning the maximum and minimum error absolute values are approximately equal to the previous iteration. Finally, the maximum percentage error in $\mathrm{z}$ is found as $0.824 \%$. The percentage error variation over the input domain is depicted in Fig. 3. The calculated construction parameters are presented in Table 2. There are two sets of solutions due to the nonlinear dependency of the construction parameters to the coefficients $\mathrm{P}_{\mathrm{j}}$ 's. A CAD model of the designed spherical 5R mechanism is constructed with the parameters in Table 2 and the function generation is confirmed.

Table 1. Calculation Results for (a)First, (b)Second, (c)Third and (d)Fourth Iteration

\begin{tabular}{|c|c|c|c|c|}
\hline & & \\
\hline
\end{tabular}




\begin{tabular}{|l|c|c|c|}
\hline \multicolumn{2}{|c|}{ Iteration 3 } & \multicolumn{2}{c|}{ Iteration 4 } \\
\hline Error $_{\min }$ & $-1.431575 \times 10^{-2}$ & Error $_{\min }$ & $-1.431586 \times 10^{-2}$ \\
\hline Error $_{\max }$ & $1.434672 \times 10^{-2}$ & Error $_{\max }$ & $1.434681 \times 10^{-2}$ \\
\hline
\end{tabular}

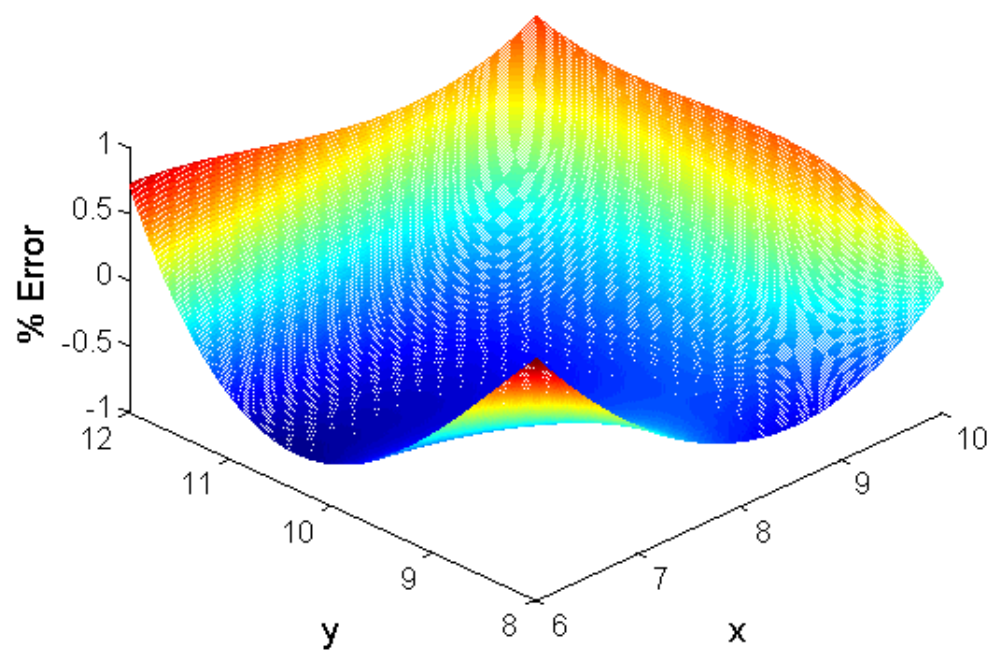

Fig. 3 Variation of percentage error in $\mathrm{z}$

Table 2. Designed construction parameter values

\begin{tabular}{|c|c|c|c|c|}
\hline$\alpha_{1}$ & $\alpha_{2}$ & $\alpha_{3}$ & $\alpha_{4}$ & $\alpha_{5}$ \\
\hline $49.971^{\circ}$ or & $159.172^{\circ}$ or & $44.336^{\circ}$ or & $26.925^{\circ}$ or & $40.3417^{\circ}$ or \\
$300.029^{\circ}$ & $339.172^{\circ}$ & $315.664^{\circ}$ & $333.075^{\circ}$ & $139.658^{\circ}$ \\
\hline
\end{tabular}

\section{Conclusions}

In this paper, the application of Chebyshev approximation method for generation of bi-variate functions using regional spacing is introduced through a case study: a 2-dof spherical 5R mechanism. First, the iterative algorithm for the approximation method is given. The synthesis problem for the spherical $5 \mathrm{R}$ mechanism is formulated by expressing the $\mathrm{I} / \mathrm{O}$ equation of the mechanism analytically for five and six link-length parameters to be determined and then the Remez algorithm for Chebyshev approximation is applied. As a numerical example, the synthesis problem with five unknown parameters is solved in order to generate the function $\mathrm{z}=\mathrm{x}^{0.45} \mathrm{y}^{0.6}$ for $6 \leq \mathrm{x} \leq 10$ and $8 \leq \mathrm{y} \leq 12$. As a result, the maximum percentage error in the generated function throughout the function domain is kept below $1 \%$.

The spherical 5R mechanism is chosen in this study as a rather simple multi-dof mechanism. In further studies we plan to apply analytical and/or semi-analytical synthesis methods to other multi-dof mechanisms with more complicated structure and also more than 2-dof.

\section{Acknowledgements}

The authors thank Prof. Rasim Alizade for his guidance. The final version of the manuscript is formed with valuable contributions of the reviewers. 


\section{References}

[1] A. Svoboda. Computing Mechanisms and Linkages, $2^{\text {nd }}$ Ed., Dover, 1965, §8-10.

[2] C. W. Allen, The design of linkages to generate function of two variables, J. Eng. Ind. 81 (1959) 23-19.

[3] R. E. Philipp, F. Freudenstein, Synthesis of two-degree-of-freedom linkages - a feasibility study of numerical methods of synthesis of bivariate function generators, J. Mechanisms 1 (1966) 9-21.

[4] K. Lakshminarayana, R. G. Narayanamurthi, Derivative synthesis of plane mechanisms to generate functions of two variables, J. Mechanisms 5 (1970) 249-71

[5] K. Lakshminarayana, A simplified approach to two-freedom linkages. J. Eng. Ind. 95 (1973) 584-588.

[6] G. Ramaiyan, K. Lakshminarayana, Contribution to synthesis of two-freedom linkages - I: Synthesis with second order precision points. ASME Paper No. 74-DET-34.

[7] K. Lakshminarayana, Contributions to the synthesis of two-freedom linkages - 2: Derivative synthesis. ASME Paper No. 74-DET-35.

[8] G. Ramaiyan, K. Lakshminarayana, Synthesis of seven-link two-freedom linkages with sliding inputs and output using identical link positions, Mech. Mach. Theory 11 (1976) 181-185.

[9] G. Ramaiyan, K. Lakshminarayana, Nine-link plane mechanisms for two-variable function generation - I. systematics, Mech. Mach. Theory 11 (1976) 187-191.

[10] G. Ramaiyan, K. Lakshminarayana, Nine-link plane mechanisms for two-variable function generation - II. synthesis, Mech. Mach. Theory 11 (1976) 193-199.

[11] T. S. Mruthyunjaya, Synthesis of plane linkages to generate functions of two variables using point position reduction part 1. rotary inputs and output. Mech. Mach. Theory 7 (1972) 363-370.

[12] T. S. Mruthyunjaya, Synthesis of plane linkages to generate functions of two variables using point position reduction II. Sliding inputs and output. Mech. Mach. Theory 7 (1972) 399-405.

[13] D. Kohli, A. H. Soni, Synthesis of seven-link mechanisms. J. Eng. Ind., 95 (1973) 533-540.

[14] R. I. Alizade, A. V. M. Rao, G. N. Sandor, Optimum synthesis of two-degree-of-freedom planar and spatial function generating mechanisms using the penalty function approach, J. Eng. Ind. 97 (1975) 629-634.

[15] A. C. Rao, Synthesis of geared planar 4-bar linkages and cams to generate functions of two variables, Mech. Mach. Theory 15 (1980) 137-143.

[16] H. Zhou, E. H. M. Cheung, Analysis and optimal synthesis of hybrid five-bar linkages, Mechatronics 11 (2001) 283 300.

[17] H. S. Kim, L-W. Tsai, Kinematic synthesis of a spatial 3-RPS parallel manipulator, J. Mech. Des. 125 (2003) 92-97.

[18] G. Kiper, B. Bağdadioğlu, T. Bilgincan, Function synthesis of the planar 5r mechanism using least squares approximation, In: J. Lenarcic, O. Khatib (Eds.), Advances in Robot Kinematics, 2014, 69-76.

[19] G. Kiper, B. Bağdadioğlu, Function generation synthesis with a 2-dof overconstrained double-spherical 7R mechanism using the method of decomposition and least squares approximation, In: P. Flores, F. Viadero, (Eds.), New Trends in Mechanism and Machine Science: From Fundamentals to Industrial Applications, Springer, 2015, 167-174.

[20] N. I. Levitskii, Synthesis of Mechanisms by Chebyshev, USSR Academy of Science, 1946.

[21] H-P. Blatt, Exchange algorithms, error estimations and strong unicity in convex programming and Chebyshev approximation, In: S. P. Singh, J. W. H. Burry (Eds.), Approximation Theory and and Spline Functions, D. Reidel Publishing Company, Dordrecht, 1983, 23-63.

[22] E. W. Cheney, Four lectures on multivariate approximation, In: S. P. Singh, J. W. H. Burry (Eds.), Approximation Theory and and Spline Functions, D. Reidel Publishing Company, Dordrecht, 1983, 65-87.

[23] V. K. Dzyadyk, I. A. Shevchuk, Theory of Uniform Approximation of Functions by Polynomials, Walter de Gruyter, Berlin, 2008, §1.

[24] Y. Kamp, J.P. Thiran, Chebyshev approximation for two-dimensional nonrecursive digital filters, IEEE Transactions on Circuits and Systems CAS-22 (1975) 208-218.

[25] G. Kiper, T. Bilgincan, M. İ. C. Dede, Function generation synthesis of planar 5R mechanism, Problems of Mechanics 2(51) (2013) 28-31.

[26] R. I. Alizade, F.C. Can, Ö. Kilit, Least square approximate motion generation synthesis of spherical linkages by using Chebyshev and equal spacing, Mech. Mach. Theory 61 (2013) 123-135.

[27] F. Freudenstein, Bi-variate, rectangular, optimum-interval interpolation, Math. Comp. 15 (1961) 288-291.

[28] J. R. Zimmerman, Four-precision synthesis of the spherical four-bar function generator, Mech. Mach. Theory 2 (1967) $133-139$.

[29] J. J. Cervantes-Sánchez, L. Gracia, J. M. Rico-Martínez, H. I. Medellín-Castillo, E. J. González-Galván, A novel and efficient kinematic synthesis approach of the spherical 4R function generator for five and six precision points, Mech. Mach. Theory 44 (2009) 2020-2037.

[30] R. I. Alizade, Ö. Kilit, Analytical synthesis of function generating spherical four-bar mechanism for the five precision points, Mech. Mach. Theory 40 (2005) 863-878.

[31] R. I. Alizade, E. Gezgin, Synthesis of function generating spherical four bar mechanism for the six independent parameters, Mech. Mach. Theory 46 (2011) 1316-1326. 
[32] F. Peñuñuri, R. Peón-Escalante, C. Villanueva, C. A. Cruz-Villar, Synthesis of spherical 4R mechanism for path generation using Differential Evolution, Mech. Mach. Theory 57 (2012) 62-70.

[33] P. V. Chanekar, M. A. A. Fenelon, A. Ghosal, Synthesis of adjustable spherical four-link mechanisms for approximate multi-path generation, Mech. Mach. Theory 70 (2013) 538-552.

[34] J. M. McCarthy, G. S. Soh. Geometric Design of Linkages, $2^{\text {nd }}$ Ed., Springer, 2010.

[35] P. L. Chebyshev, Théorie des mécanismes connus sous le nom de parallélogrammes. Mémoires Présentés à L'académie Impériale des Sciences de St.-Pétersbourg par Divers Savants 7 (1854) 539-568.

[36] P. L. Chebyshev, Sur les questions de minima qui se rattachent à la representation approximative des fonctions. Mémoires Présentés à L'académie Impériale des Sciences de St.-Pétersbourg, Sciences Mathématiques et Physiques 7 (1859) 199-291.

[37] E. Remez,. Sur le calcul effectif des polynomes d'approximation de Tschebyscheff. C. R. Acad. Sci. Paris199 (1934) 337-340.

[38] G.C. Temes, Iterative Chebyshev approximation technique for network synthesis, IEEE Trans. Circuit Theory 14 (1967) 31-37. 